\title{
Reevaluation of a Tetraploid Wheat Population Indicates that the Tsn1-ToxA Interaction Is the Only Factor Governing Stagonospora nodorum Blotch Susceptibility
}

\author{
Justin D. Faris and Timothy L. Friesen
}

United States Department of Agriculture-Agricultural Research Service Cereal Crops Research Unit, Northern Crop Science Laboratory, 1307 18th Street North, Fargo, ND 58105.

Accepted for publication 7 April 2009.

\section{ABSTRACT}

Faris, J. D., and Friesen, T. L. 2009. Reevaluation of a tetraploid wheat population indicates that the Tsn1-ToxA interaction is the only factor governing Stagonospora nodorum blotch susceptibility. Phytopathology 99:906-912.

The wheat $T s n 1$ gene on chromosome 5B confers sensitivity to a hostselective toxin produced by the pathogens that cause tan spot and Stagonospora nodorum blotch (SNB) known as Ptr ToxA and SnToxA, respectively (hereafter referred to as ToxA). A compatible Tsn1-ToxA interaction is known to play a major role in conferring susceptibility of hexaploid (common) wheat to SNB. However, a recent study by another group suggested that the Tsn1-ToxA interaction was not relevant in conferring susceptibility of the tetraploid (durum) wheat cv. Langdon (LDN). Here, we reevaluated the role of the Tsn1-ToxA interaction in governing SNB susceptibility using the same mapping population and
Stagonospora nodorum isolate ( $\mathrm{Sn2000)}$ as were used in the previous study. Results of our quantitative trait locus analysis showed that the Tsn1 locus accounted for $95 \%$ of the variation in SNB. In addition, inoculation of the mapping population with two ToxA-knockout strains of Sn2000 revealed that the entire population was resistant. Furthermore, several LDN Tsn1-disrupted mutants were evaluated and found to be resistant to SNB. Together, these results prove unequivocally that $T s n 1$ is the only factor present along chromosome 5B that governs response to SNB in this population and that a compatible Tsn1-ToxA interaction is necessary for the manifestation of disease. Therefore, the results from the previous study are refuted.

Additional keywords: Phaeosphaeria nodorum, resistance, segregation distortion.
Stagonospora nodorum blotch (SNB) caused by Stagonospora nodorum (Berk.) E. Castell. \& Germano (anamorph; Phaeosphaeria nodorum) and tan spot caused by Pyrenophora triticirepentis (Died.) Drechsler (anamorph; Drechslera tritici-repentis (Died.) Shoemaker) are both devastating foliar diseases of hexaploid common wheat (Triticum aestivum L., $2 \mathrm{n}=6 \mathrm{x}=42$, AABBDD genomes) and tetraploid durum wheat (T. turgidum L., $2 \mathrm{n}=4 \mathrm{x}=28$, AABB genomes). Both diseases are caused by necrotrophic pathogens that occur in all major wheat-growing areas of the world, and they have the capacity to cause significant yield losses $(6,19)$. In addition, $S$. nodorum can infect wheat heads causing glume blotch, which can severely impact grain quality (7).

Most studies have indicated that resistance to SNB in wheat is quantitatively inherited (35). However, there is rapidly accumulating evidence for the wheat-S. nodorum pathosystem being a toxin-based inverse gene-for-gene system $(11,13,14,25-27)$. In the wheat $-S$. nodorum system, a compatible interaction results when a specific host-selective toxin (HST) is recognized directly

Corresponding author: J. D. Faris; E-mail address: Justin.Faris@ ars.usda.gov

Mention of trade names or commercial products in this article is solely for the purpose of providing specific information and does not imply recommendation or endorsement by the United States Department of Agriculture.

* The $e$-Xtra logo stands for "electronic extra" and indicates that Figures 1 and 2 appear in color online.

doi:10.1094/PHYTO-99-8-0906

This article is in the public domain and not copyrightable. It may be freely reprinted with customary crediting of the source. The American Phytopathological Society, 2009. or indirectly by a specific dominant gene in the host. This recognition leads to the manifestation of extensive cell death, and disease ensues. Incompatible interactions result when either an HST is not produced by the pathogen or the corresponding dominant host gene is not present. In this case, recognition between the host and pathogen does not occur and, therefore, results in resistance. The segregation of multiple toxin sensitivity genes in a given population leads to the perception that resistance is governed quantitatively.

To date, four $S$. nodorum-produced HSTs (SnTox1, SnTox2, SnTox3, and SnToxA) and their corresponding host sensitivity genes (Snn1, Snn2, Snn3, and Tsn1) have been reported $(8,11$, 13,14,25,26). Sensitivity to the HSTs SnTox1, SnTox2, and SnTox 3 is governed by the dominant host genes Snn1, Snn2, and Snn3, which have been mapped to wheat chromosome arms 1BS, 2DS, and 5BS, respectively $(11,14,25)$. In each case, compatible interactions were shown to play significant roles in disease development.

Compared with the Snn1-SnTox1, Snn2-SnTox2, and Snn3SnTox 3 interactions, more is known about the Tsn1-ToxA interaction, which was first described in the wheat $-P$. tritici repentis system. Necrosis-inducing isolates of $P$. tritici-repentis are known to release the proteinaceous host-selective toxin Ptr ToxA in culture, which causes cell death in leaves of sensitive wheat genotypes $(21,33)$. Early work showed that production of the toxin was associated with pathogenicity $(5,34)$, and host sensitivity to the toxin was associated with susceptibility to the fungus $(21,23)$ and disease severity (12). Faris et al. (8) identified a single dominant gene $(T s n 1)$ on the long arm of chromosome $5 \mathrm{~B}$ conditioning sensitivity to necrosis caused by Ptr ToxA. More recently, Friesen et al. (13) showed that $S$. nodorum also possessed the ToxA gene 
and that it was horizontally transferred to $P$. tritici-repentis. They showed that the $S$. nodorum ToxA protein was functionally identical to Ptr ToxA and that it was an important factor in conferring SNB. Therefore, they designated the $S$. nodorum ToxA protein as SnToxA. Liu et al. (26) used a common wheat intervarietal mapping population and Tsn1-disrupted mutants to demonstrate that Tsnl confers sensitivity to both Ptr ToxA and SnToxA. A compatible Tsn1-ToxA interaction was shown to account for as much as $68 \%$ of the variation in disease, indicating that this interaction plays a highly significant role in the development of SNB.

Gonzalez-Hernandez et al. (15) evaluated a population of recombinant inbred chromosome lines (RICLs) derived from the durum cv. Langdon (LDN) and an LDN-T. turgidum subsp. dicoccoides chromosome 5B disomic substitution line (LDN-DIC 5B) for reaction to SNB caused by the isolate $\mathrm{Sn} 2000$. They reported a quantitative trait locus (QTL) on chromosome 5B 8.8 centimorgans (cM) proximal to the Tsn1 locus that explained $37.6 \%$ of the variation in disease. Although we had not investigated the effects of the Tsnl locus and a compatible Tsn1-ToxA interaction on SNB development in a tetraploid wheat background, we found it interesting that Gonzalez-Hernandez et al. (15) reported no significant effects for the $T s n 1$ locus in association with susceptibility to SNB. Therefore, the objective of this work was to reevaluate the effects of $T_{s n 1}$ on SNB susceptibility in tetraploid wheat using the same mapping population and fungal isolate (Sn2000) as was used by Gonzalez-Hernandez et al. (15). In addition, we employed LDN Tsnl-disrupted mutants and Sn2000 ToxA-knockout fungal isolates to further investigate whether or not different genes conferring susceptibility to SNB reside on LDN chromosome 5B.

\section{MATERIALS AND METHODS}

Plant materials. We evaluated the 85 RICLs derived from LDN and LDN-DIC 5B (hereafter referred to as the LD5B population) that Faris et al. (9) used to generate a partial linkage map of chromosome 5B. This population was developed by Dr. Leonard Joppa (United States Department of Agriculture-Agricultural Research Service [USDA-ARS], retired) and is maintained by the USDA-ARS Cereal Crops Research Unit, Fargo, ND. The generation of the RICL population was described by Joppa (18). For this experiment, we used original seed maintained and increased by Dr. Joppa. It is important to note that this population segregates only for chromosome $5 \mathrm{~B}$ and the rest of the background is homozygous for LDN chromosomes.

Five independent ToxA-insensitive mutants, designated LDNems138, LDNems299, LDNems355, LDNems403, and LDNems937, were generated in the LDN background using the methods described by Simons et al. (32). Validation that the mutants harbored a disrupted Tsnl gene was carried out as described by Liu et al. (26) using the markers described by Lu et al. (29) and Zhang et al. (37).

ToxA bioassays. ToxA was produced and purified from the $P$. tritici-repentis race 2 isolate 86-124 (22) as described by Zhang et al. (36). The mutants, parents, and RICLs were infiltrated with $\approx 25 \mu \mathrm{l}$ of purified ToxA at the second leaf stage as described by Liu et al. (26). Infiltrated leaves were evaluated 4 days after infiltration and scored as insensitive or sensitive. Sensitive reactions were characterized by the presence of necrosis throughout the area of infiltration and insensitive reactions showed no visible necrosis. This experiment consisted of three replications.

Inoculum preparation and disease screening. Conidial inoculations were done as described by Friesen et al. (13) with slight modifications. The LD5B population along with parental lines LDN and LDN-DIC 5B were planted in racks consisting of 98 cones (Steuwe \& Sons, Corvallis, OR), including a complete border of the SNB susceptible hard red spring wheat cv. Grandin to eliminate any edge effect. The 85 lines and parents were each planted at a rate of three seeds per line per cone. Conidia were produced and harvested as described by Liu et al. (27). Plants were inoculated using $1 \times 10^{6}$ spores $/ \mathrm{ml}$ and inoculated until runoff. Following inoculation, plants were placed in $100 \%$ relative humidity at $21^{\circ} \mathrm{C}$ in the light for $24 \mathrm{~h}$ followed by 6 days at $21^{\circ} \mathrm{C}$ under a 12-h photoperiod. The three plants of each line were evaluated collectively at 7 days postinoculation using the 0 to 5 scale described by Liu et al. (27), where 0 is highly resistant and 5 is highly susceptible. Lines were evaluated blindly so as not to bias the outcome. Three replicates were completed for each isolate or strain tested.

Genetic mapping. Restriction fragment length polymorphism (RFLP) markers were used for generating a genetic linkage map of chromosome $5 \mathrm{~B}$ in the LD5B population. DNA isolation, restriction enzyme digestion, gel electrophoresis, probe preparation and labeling, Southern blotting, hybridization, and membrane washing were done as described by Faris et al. (9). RFLP probes known to detect wheat chromosome $5 \mathrm{~B}$ fragments were selected from the following libraries: barley genomic (ABG and $\mathrm{MWG}$ ), barley cDNA (BCD), oat cDNA (CDO), rice cDNA (RZ), and wheat genomic (FCG, KSU, PSR, and WG). Linkage analysis was conducted using Mapmaker v2.0 for Macintosh (24) as described by Faris et al. (9).

Statistical analysis. Segregation ratios of the 85 RICLs for reaction to ToxA and for marker genotypes were tested for fit to a $1: 1$ ratio by $\chi^{2}$ analysis using the computer program QGene v.3.06 (31). Simple and composite interval mapping was conducted using the disease reaction type means of the LD5B population for each isolate using MapManager QTX (30) and QGene v.4.0 (17). A critical log of the likelihood ratio (LOD) threshold of 1.8 was established by executing a permutation test with 1,000 permutations.

\section{RESULTS}

ToxA infiltrations. Infiltration of the LD5B population with ToxA gave clear, unambiguous results, just as has been previously observed in related research $(13,16,26)$. The Tsn1-disrupted mutants were all insensitive to ToxA, as expected. LDN was sensitive to ToxA and LDN-DIC 5B was insensitive. The LD5B population segregated in a ratio of 30:55 insensitive/sensitive, which did not fit the expected 1:1 ratio (Table 1). By definition, reaction to ToxA indicates the Tsn1 genotype. Therefore, the reactions of the RICLs to ToxA were assigned genotypic values and used as a phenotypic marker (Tsnl) in chromosome 5B linkage analysis.

Chromosome 5B linkage mapping. In total, 28 markers, including 27 RFLPs and Tsn1, were used to assemble the genetic linkage map of chromosome 5B in the LD5B population (Fig. 1). The map spanned a genetic distance of $102.2 \mathrm{cM}$. Markers $X b c d 183$ and $X r z 575$ flanked the Tsnl locus at distances of 1.2 and $2.4 \mathrm{cM}$, respectively, and $T s n 1$ cosegregated with the marker Xbcd1030.

Analysis of RFLP marker segregation ratios indicated that 22 of the 27 markers had skewed segregation ratios (Table 1). In each case, ratios were skewed in favor of LDN alleles and there were fewer than expected LDN-DIC 5B alleles. Only the five most distal markers on the long arm had ratios that conformed to the expected 1:1 ratio. This result agrees with the fact that, along chromosome $5 \mathrm{~B}$, there are factors that cause preferential transmission of male gametes harboring LDN alleles over gametes harboring alleles of the T. turgidum subsp. dicoccoides accession used to generate LDN-DIC 5B (20).

Reaction to SNB caused by $\boldsymbol{S}$. nodorum isolates. The LDN Tsn1-disrupted mutants, LDN, LDN-DIC 5B, and the LD5B population were evaluated for reaction to SNB caused by $S$. nodorum isolates $\mathrm{Sn} 2000$ and two strains (Sn2000KO6-1 and Sn2000KO28) of Sn2000 that have had the ToxA gene eliminated 
and, thus, do not produce ToxA (13). For reaction to the Sn2000 wild-type strain, LDN was susceptible, with an average reaction type of 3.5, whereas LDN-DIC 5B was resistant, with an average reaction type of 0.8 (Table 2; Fig. 2). Average reaction types of the LD5B population were 0.3 to 3.8 with an overall average of 2.4. The distribution of Sn2000 reaction types resembled a bimodal distribution suggesting that, in the LD5B population, a single gene governs resistance to SNB caused by Sn2000 (Fig. 3).

Gonzalez-Hernandez et al. (15) reported that several lines, including RICLs 4, 5, and 46, were susceptible to SNB but insensitive to ToxA, and that RICLs 63 and 89 were resistant to SNB but sensitive to ToxA. Our data agree with Gonzalez-Hernandez et al. (15) in that RICLs 4, 5, and 46 are insensitive to ToxA and RICLs 63 and 89 are sensitive. However, our data disagree in that RICLs 4, 5, and 46 were resistant to Sn2000 and 63 and 89 were susceptible (Table 3; Fig. 2).

Simple interval regression mapping of $\mathrm{Sn} 2000$ reaction types indicated a QTL peaking at the Tsn1 locus on chromosome 5B associated with resistance to SNB caused by Sn2000 (Fig. 1). The QTL had a maximum LOD value of 54.0 and explained $95 \%$ of the variation in disease caused by $\mathrm{Sn} 2000$. Similarly, composite interval mapping of the same data set demonstrated that only one QTL was present on chromosome 5B, and the Tsn1 locus defined the peak of the QTL, explaining 95\% of the variation.

LDN, LDN-DIC 5B, and all 85 RICLs were resistant to inoculations with the ToxA knockout isolates Sn2000KO6-1 and Sn2000KO28 (Table 2). Average reaction types for LDN and LDN-DIC 5B were 1.2 and 0.5 for Sn2000KO6-1 and 0.5 and 0.8 for $\mathrm{Sn} 2000 \mathrm{KO} 28$, respectively. Average reaction types for the LD5B population were 0.7 and 0.6 for Sn2000KO6-1 and Sn2000KO28, respectively, and reaction type ranges in the LD5B population were 0 to 1.2 and 0 to 1.3 , respectively.

Inoculation of the LDN Tsn1-disrupted mutants with Sn2000, Sn2000KO6-1, and Sn2000KO28 indicated that all were resistant (Table 2), with mean reaction types of 0.2 to 1.0 for the three isolates. Together, these results demonstrate that $T s n 1$ is the only factor on LDN chromosome 5B governing susceptibility to SNB caused by isolate $\mathrm{Sn} 2000$, and they show that a compatible Tsn1ToxA interaction is necessary for the development of SNB in the LD5B population.

\section{DISCUSSION}

The Tsnl gene, which confers sensitivity to the HST ToxA produced by both the $S$. nodorum and the $P$. tritici repentis pathogens, is known to play a major role in conferring susceptibility to ToxA-producing isolates of $P$. tritici-repentis and $S$. nodorum in hexaploid wheat $(1,3,11-14,21,23)$. Although we had not previously conducted direct studies to evaluate the role of Tsnl in conferring susceptibility in tetraploid wheat backgrounds, Chu et al. $(2,4)$ reported statistical associations between ToxA sensitivity and susceptibility to SNB in accessions of various tetraploid subspecies. Therefore, we found it peculiar that Gonzalez-Hernandez et al. (15) reported that $T_{s n} 1$ had no effect on susceptibility to SNB in the LD5B population and, instead, suggested that a QTL $8.8 \mathrm{cM}$ proximal to $T s n 1$ was responsible for governing reaction to SNB caused by isolate $\mathrm{Sn} 2000$.

In an effort to validate the results of Gonzalez-Hernandez et al. (15), we reevaluated the same population for reaction to SNB caused by $\mathrm{Sn} 2000$, the same isolate used by Gonzalez-Hernandez et al. (15), and constructed our own genetic linkage map of chromosome $5 \mathrm{~B}$ in this population. Furthermore, we inoculated the population with two Sn2000 strains that harbor a disrupted ToxA gene (13) to further evaluate the effects of ToxA in conferring SNB susceptibility. In line with this, we included our LDN Tsn1-disrupted mutants in all inoculation experiments to further solidify our findings regarding the role of a compatible Tsn1ToxA interaction in conferring disease in the LD5B population.

In contrast to Gonzalez-Hernandez et al. (15), our results clearly demonstrate that $T s n 1$ is the one and only factor segregating on chromosome $5 \mathrm{~B}$ in the LD5B population that governs ToxA sensitivity and reaction to $\mathrm{SNB}$. The various lines of evidence include the following. First, all RICLs that were sensitive to ToxA were also susceptible to SNB caused by Sn2000, indi-

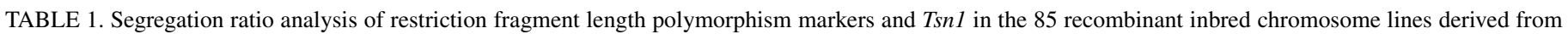
wheat cv. Langdon $(\mathrm{LDN}) \times \mathrm{LDN}-\mathrm{DIC} 5 \mathrm{~B}$

\begin{tabular}{|c|c|c|c|c|c|}
\hline Marker & Position & $n$ & Observed LDN:LDN-DIC 5B allelic ratios ${ }^{\mathrm{z}}$ & $\chi^{2}$ & $P$ \\
\hline Xbcd873 & 0.0 & 83 & $59: 24$ & 14.75 & 0.0001 \\
\hline Xabg705 & 13.6 & 85 & $61: 24$ & 16.10 & 0.0001 \\
\hline Xbcd1871 & 13.6 & 84 & $60: 24$ & 15.42 & 0.0001 \\
\hline Xwg363 & 26.8 & 85 & $64: 21$ & 21.75 & $<0.0001$ \\
\hline XksuA3 & 26.8 & 85 & $64: 21$ & 21.75 & $<0.0001$ \\
\hline$X b c d 204$ & 26.8 & 62 & $46: 16$ & 14.51 & 0.0001 \\
\hline Xpsr128 & 27.4 & 82 & $62: 20$ & 21.51 & $<0.0001$ \\
\hline Xbcd157 & 28.0 & 82 & $61: 21$ & 19.51 & $<0.0001$ \\
\hline XksuH1 & 31.5 & 83 & $61: 22$ & 18.32 & $<0.0001$ \\
\hline$X b c d 1140$ & 31.5 & 85 & $62: 23$ & 17.89 & $<0.0001$ \\
\hline$X f c g 3$ & 33.9 & 83 & $60: 23$ & 16.49 & $<0.0001$ \\
\hline Xmwg914 & 42.2 & 82 & $56: 26$ & 10.97 & 0.0009 \\
\hline Xmwg72 & 43.4 & 85 & $56: 29$ & 8.57 & 0.0034 \\
\hline$X k s u Q 63$ & 43.4 & 81 & $55: 26$ & 10.38 & 0.0013 \\
\hline Xpsr120 & 44.0 & 85 & $56: 29$ & 8.57 & 0.0034 \\
\hline$X b c d 9$ & 44.6 & 83 & $56: 27$ & 10.13 & 0.0015 \\
\hline Xwg583 & 44.6 & 85 & $57: 28$ & 9.89 & 0.0017 \\
\hline Xcdo400 & 45.8 & 84 & $56: 28$ & 9.33 & 0.0023 \\
\hline Xbcd183 & 47.0 & 81 & $55: 26$ & 10.38 & 0.0013 \\
\hline Tsn1 & 48.2 & 85 & $55: 30$ & 7.36 & 0.0067 \\
\hline Xbcd1030 & 48.2 & 85 & $55: 30$ & 7.36 & 0.0067 \\
\hline Xrz.575 & 50.6 & 82 & $55: 27$ & 9.56 & 0.0020 \\
\hline Xcdo948 & 63.0 & 84 & $53: 31$ & 5.76 & 0.0164 \\
\hline$X f c g 4$ & 72.9 & 84 & $49: 35$ & 2.33 & 0.1269 \\
\hline$X f c g 28(Q)$ & 86.6 & 84 & $45: 39$ & 0.42 & 0.5169 \\
\hline Xpsr370 & 89.3 & 84 & $48: 36$ & 1.71 & 0.1910 \\
\hline$X m w g 862$ & 96.1 & 84 & $48: 36$ & 1.71 & 0.1910 \\
\hline Xpsr580 & 102.2 & 82 & $42: 40$ & 0.04 & 0.8415 \\
\hline
\end{tabular}

${ }^{\mathrm{z}}$ Expected ratio of LDN:LDN-DIC 5B alleles is 1:1. 
cating perfect correlation between sensitivity and insensitivity to ToxA and susceptibility and resistance to SNB. This result is in contrast to that reported by Gonzalez-Hernandez et al. (15), in that they reported several RICLs to be insensitive to ToxA but susceptible to SNB, and vice versa. Specifically, two ToxA-sensitive lines (lines 63 and 89) were reported to be resistant to the ToxA-producing Sn2000 and three ToxA-insensitive lines (lines 4,5 , and 46) were reported to be susceptible to $\mathrm{Sn} 2000$.
Second, our analysis revealed a single massive QTL on chromosome 5B associated with reaction to SNB that peaked at the Tsn1 locus and explained $95 \%$ of the variation in the development of SNB. Gonzalez-Hernandez et al. (15) reported no significant effects of the Tsn1 locus associated with SNB. Instead, they reported a relatively minor QTL $8.8 \mathrm{cM}$ proximal to $T s n 1$ that explained $37.6 \%$ of the variation. They attributed their relatively low $R^{2}$ value to two possible explanations. First, they mention that

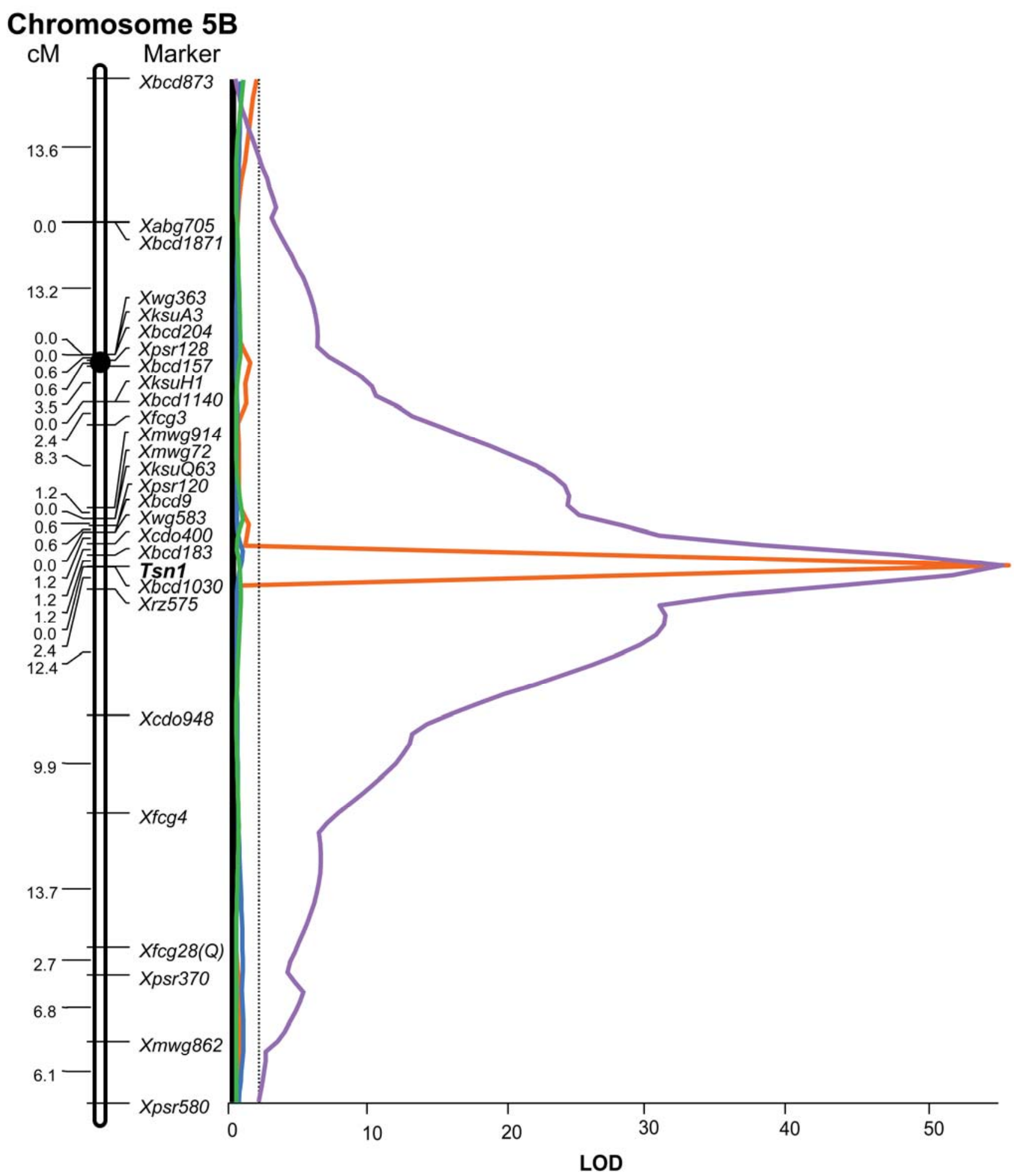

Fig. 1. Genetic linkage map of chromosome 5B (left) and quantitative trait locus analysis (right) of the average reaction types of Stagonospora nodorum isolate Sn2000 using simple interval regression (purple) and composite interval regression (orange). Average reaction types of the Sn2000 ToxA-knockout strains Sn2000KO6-1 and Sn2000KO28 were analyzed using simple interval regression and are shown in green and blue, respectively. The dotted line represents the critical $\log$ of the likelihood ratio threshold of 1.8 . 


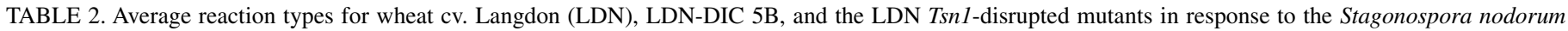
isolates Sn2000, Sn2000KO6-1, and Sn2000KO28

\begin{tabular}{|c|c|c|c|c|c|c|c|c|}
\hline \multirow[b]{3}{*}{ Genetic stock } & \multirow[b]{3}{*}{ Genotype $^{\mathrm{x}}$} & \multirow[b]{3}{*}{$\operatorname{ToxA}^{\mathrm{y}}$} & \multicolumn{6}{|c|}{ Isolate $^{\mathrm{z}}$} \\
\hline & & & \multicolumn{2}{|c|}{$\operatorname{Sn} 2000$} & \multicolumn{2}{|c|}{ Sn2000KO6-1 } & \multicolumn{2}{|c|}{$\mathrm{Sn} 2000 \mathrm{KO} 28$} \\
\hline & & & RT & Range & RT & Range & $\mathrm{RT}$ & Range \\
\hline LDN & $T s n 1 / T s n 1$ & + & $3.5 \mathrm{~b}$ & $3.0-4.0$ & $1.2 \mathrm{a}$ & $0.5-1.5$ & $0.5 \mathrm{a}$ & $0.0-1.0$ \\
\hline LDN-DIC 5B & $t s n 1 / t \operatorname{sn} 1$ & - & $0.8 \mathrm{a}$ & $0.5-1.0$ & $0.5 \mathrm{a}$ & $0.0-1.0$ & $0.8 \mathrm{a}$ & $0.5-1.0$ \\
\hline LDNems 138 & $T s n 1^{m} / T s n 1^{m}$ & - & $0.5 \mathrm{a}$ & $0.0-1.0$ & $0.5 \mathrm{a}$ & $0.0-1.0$ & $0.7 \mathrm{a}$ & $0.0-1.0$ \\
\hline LDNems 299 & $T s n 1^{m} / T s n 1^{m}$ & - & $0.2 \mathrm{a}$ & $0.0-0.5$ & $0.7 \mathrm{a}$ & $0.5-1.0$ & $0.5 \mathrm{a}$ & $0.5-0.5$ \\
\hline LDNems 355 & $T s n 1^{m} / T s n 1^{m}$ & - & $1.0 \mathrm{a}$ & $1.0-1.0$ & $0.8 \mathrm{a}$ & $0.0-1.5$ & $1.0 \mathrm{a}$ & $0.5-1.5$ \\
\hline LDNems403 & $T s n 1^{m} / T s n 1^{m}$ & - & $0.8 \mathrm{a}$ & $0.5-1.0$ & $0.5 \mathrm{a}$ & $0.0-1.0$ & $0.8 \mathrm{a}$ & $0.0-1.5$ \\
\hline LDNems937 & $T s n 1^{m} / T s n 1^{m}$ & - & $0.8 \mathrm{a}$ & $0.5-1.0$ & $0.7 \mathrm{a}$ & $0.0-1.0$ & $0.8 \mathrm{a}$ & $0.5-1.0$ \\
\hline LD5B population & $\ldots$ & $\ldots$ & 2.4 & $0.3-3.8$ & 0.7 & $0.0-1.2$ & 0.6 & $0.0-1.3$ \\
\hline
\end{tabular}

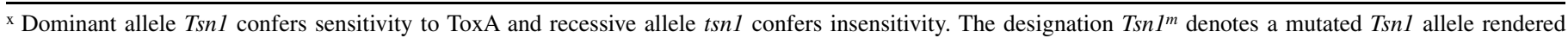
nonfunctional.

y Sensitive (+) and insensitive (-) to ToxA.

${ }^{\mathrm{z}} \mathrm{RT}=$ reaction type average; numbers within the same column followed by the same letters are not significantly different at the 0.05 level of probability.

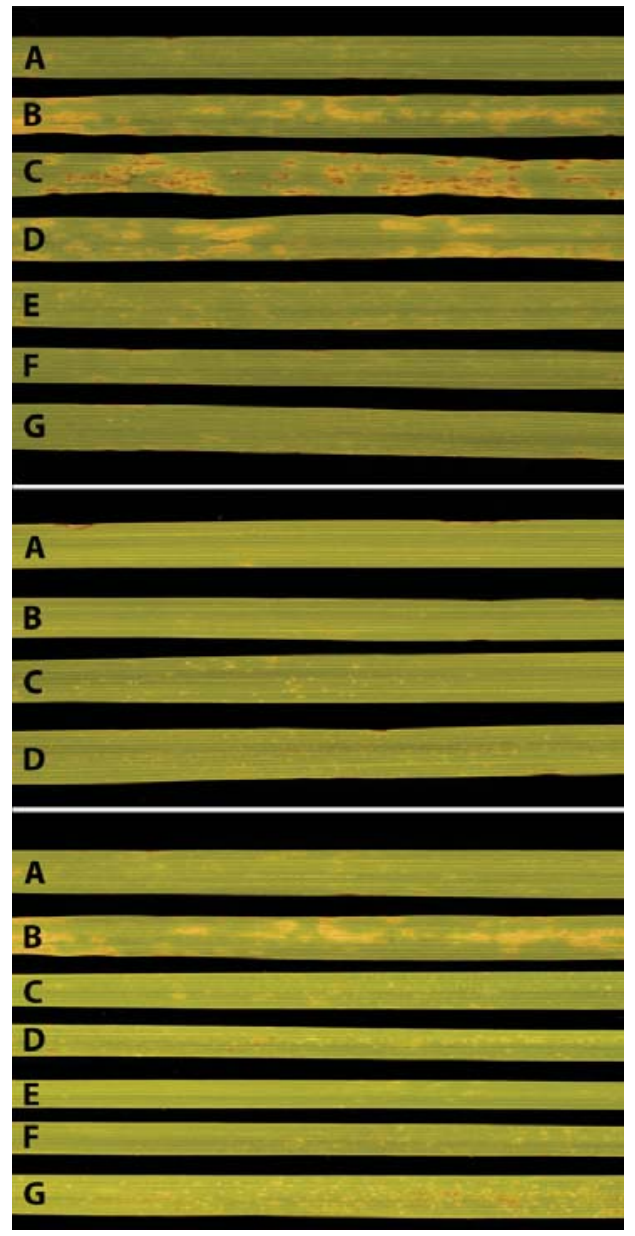

Fig. 2. Reaction types of wheat cv. Langdon (LDN), LDN-DIC 5B, several LDN5B recombinant inbred chromosome lines (RICLs), and the Tsn1disrupted mutants with Stagonospora nodorum isolate Sn2000 and the ToxAknockout strain Sn2000KO28. Top panel: leaves inoculated with Sn2000. Line A, LDN-DIC 5B; line B, LDN; line C, RICL no. 63; line D, RICL no. 89; line E, RICL no. 4; line F, RICL no. 5; and line G, RICL no. 46. Middle panel: leaves inoculated with Sn2000KO28. Line A, LDN; line B, LDN-DIC 5B; line C, RICL no. 89; and line D, RICL no. 63. Bottom panel: Tsn1-disrupted mutants inoculated with Sn2000. Line A, LDN-DIC 5B; line B, LDN; line C, LDNems937; line D, LDNems355; line E, LDNems138; line F, LDNems403; and line G, LDNems299.

the 1 to 5 rating scale (10) that they used limits the range of values that can be given to each plant, which can reduce the precision of measurement and underestimate the true effects of the QTL. Secondly, Gonzalez-Hernandez et al. (15) suggested

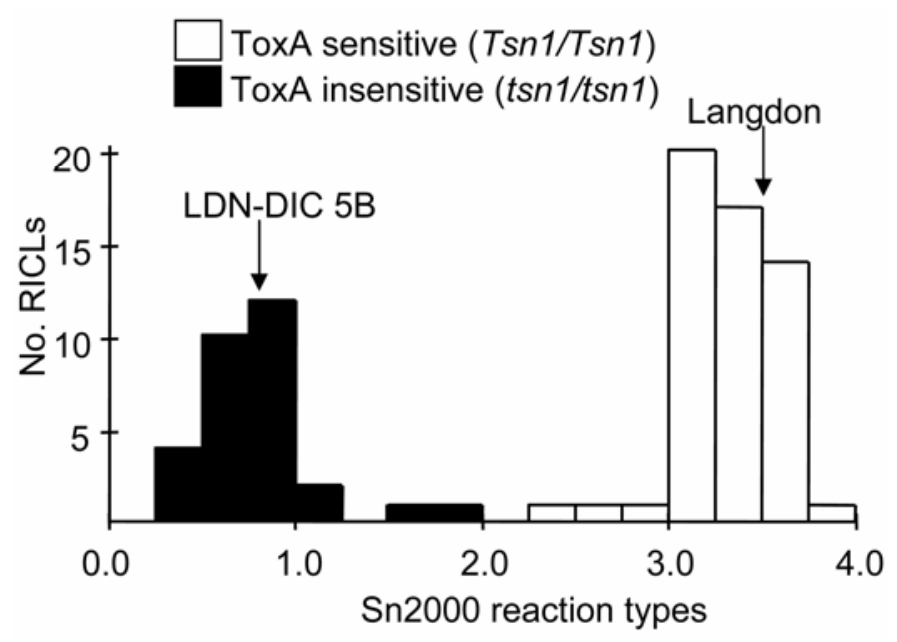

Fig. 3. Histograms of average Stagonospora nodorum blotch reaction types observed in the LD5B population caused by Stagonospora nodorum isolate Sn2000. RICL = recombinant inbred chromosome line.

that the segregation distortion observed in the vicinity of the QTL can lead to an underestimation of the effect of the QTL. In our work, we used a similar scale based on lesion type ratings of 0 to 5 (27), and we observed the severe segregation distortion of marker ratios just as they did; however, we demonstrated that the $T s n 1$ locus explained nearly all of the phenotypic variation with an $R^{2}$ value for the average SNB reaction types of 0.95 . Therefore, the two reasons projected by Gonzalez-Hernandez et al. (15) for achieving a low $R^{2}$ value are unsuitable explanations.

Finally, our data obtained from screening the RICL population with the Sn2000 ToxA-knockout strains and inoculation of the LDN Tsn1-disrupted mutants demonstrated unequivocally that Tsn1 is the only factor on chromosome 5B in the LD5B population that governs susceptibility to SNB caused by Sn2000. Inoculation of the RICLs with Sn2000 ToxA-knockouts revealed that all were resistant to these strains and, thus, the QTL observed at the $T_{s n 1}$ locus with the wild-type $\mathrm{Sn} 2000$ isolate was not present. Furthermore, the fact that the LDN Tsn1-disrupted mutants were all resistant to isolate $\mathrm{Sn} 2000$ proves that $T s n 1$ is the only factor necessary for conferring susceptibility in the LD5B population.

Although there are only eight markers in common between the map generated by Gonzalez-Hernandez et al. (15) and our map, there are some obvious differences. For example, markers $X b c d 873$ and $X a b g 705$ are separated by $2.1 \mathrm{cM}$ on the GonzalezHernandez et al. (15) map and by $13.6 \mathrm{cM}$ on our map. Also, Tsn1 and $\mathrm{Xbcd1030}$ cosegregated on our map but were separated by 2.7 
TABLE 3. Responses of five recombinant inbred chromosome lines (RICLs) to ToxA and the Stagonospora nodorum isolate Sn2000 that were reported by Gonzalez-Hernandez et al. (15) to have differential responses to ToxA and Stagonospora nodorum blotch (SNB) caused by Sn2000

\begin{tabular}{|c|c|c|c|c|c|c|}
\hline \multirow[b]{2}{*}{ Line } & \multicolumn{3}{|c|}{ Gonzalez-Hernandez et al. (15) } & \multicolumn{3}{|c|}{ Current study } \\
\hline & $\operatorname{Tox}^{\mathrm{z}}$ & SNB rating & SNB reaction & $\operatorname{Tox}^{\mathrm{z}}$ & SNB rating & SNB reaction \\
\hline RICL no. 63 & + & 1.86 & Resistant & + & 3.8 & Susceptible \\
\hline RICL no. 89 & + & 2.07 & Resistant & + & 3.7 & Susceptible \\
\hline RICL no. 4 & - & 3.55 & Susceptible & - & 0.8 & Resistant \\
\hline RICL no. 5 & - & 3.47 & Susceptible & - & 0.8 & Resistant \\
\hline RICL no. 46 & - & 3.25 & Susceptible & - & 0.3 & Resistant \\
\hline
\end{tabular}

${ }^{z}$ Gonzalez-Hernandez et al. (15) labeled this column as "Tan spot reaction" but reactions to tan spot were not evaluated in their work; instead, they were referring to response to ToxA infiltrations. Here, $+=$ sensitive and $-=$ insensitive to ToxA.

$\mathrm{cM}$ on their map. Markers $X w g 583$ and $\mathrm{Xbcd9}$, which are common to both maps and were reported by Gonzalez-Hernandez et al. (15) to be significantly associated with SNB, were more than $8 \mathrm{cM}$ proximal to $T s n 1$ on their map but only $3.6 \mathrm{cM}$ proximal to Tsnl on our map.

We cannot define the exact reasons for the discrepant results of the Gonzalez-Hernandez et al. (15) study. Regardless, it was necessary for us to perform this reevaluation in order to preserve the integrity and correctness of information regarding the role of the $T$ sn 1-ToxA interaction in the wheat-S. nodorum pathosystem. Most importantly, Gonzalez-Hernandez et al. (15) claim that, because they found apparent recombination between Tsn1 and an SNB resistance QTL on chromosome 5B, different loci confer susceptibility to SNB and sensitivity to Ptr ToxA. Our results demonstrate that $T_{s n 1}$ itself is responsible for sensitivity to ToxA produced by both $P$. tritici-repentis and $S$. nodorum and, therefore, is the only locus governing toxin sensitivity and SNB susceptibility in this population.

This work substantiates the role of Tsnl in conferring SNB susceptibility in a tetraploid wheat background. In the process of cloning Tsnl using a map-based approach, we have developed saturated and high-resolution genetic linkage maps of the Tsnl locus $(16,28,29)$, developed user-friendly high-throughput markers suitable for marker-assisted selection $(29,37)$, and isolated the Tsn1 gene (J. D. Faris, unpublished). The isolation of Tsn1 will allow us to unravel the molecular mechanisms underlying Tsn1ToxA interactions and provide further knowledge regarding the wheat-S. nodorum inverse gene-for-gene system that involves both tetraploid and hexaploid wheat hosts.

\section{ACKNOWLEDGMENTS}

This research was supported by USDA-ARS CRIS projects 544221000-033 and 5442-22000-037.

\section{LITERATURE CITED}

1. Cheong, J., Wallwork, H., and Williams, K. J. 2004. Identification of a major QTL for yellow leaf spot resistance in the wheat varieties Brookton and Cranbrook. Aust. J. Agric. Res. 55:315-319.

2. Chu, C.-G., Friesen, T. L., Faris, J. D., and Xu, S. S. 2008. Evaluation of seedling resistance to tan spot and Stagonospora nodorum blotch in tetraploid wheat. Crop Sci. 48:1107-1116.

3. Chu, C.-G., Friesen, T. L., Xu, S. S., and Faris, J. D. 2008. Identification of novel tan spot resistance loci beyond the known host-selective toxin insensitivity genes in wheat. Theor. Appl. Genet. 117:873-881.

4. Chu, C.-G., Xu, S. S., Faris, J. D., Nevo, E., and Friesen, T. L. 2008. Seedling resistance to tan spot and Stagonospora nodorum leaf blotch in wild emmer wheat (Triticum dicoccoides). Plant Dis. 92:1229-1236.

5. Ciuffetti, L. M., Tuori, R. P., and Gaventa, J. M. 1997. A single gene encodes a selective toxin causal to the development of tan spot of wheat. Plant Cell 9:135-144.

6. Eyal, Z. 1981. Integrated control of Septoria diseases of wheat. Plant Dis. 65:763-768.

7. Eyal, Z., Scharen, A. L., Prescott, J. M., and van Ginkel, M. 1987. The Septoria Diseases of Wheat: Concepts and Methods of Disease Management. CIMMYT, Mexico, D.F.

8. Faris, J. D., Anderson, J. A., Francl, L. J., and Jordahl, J. G. 1996. Chromosomal location of a gene conditioning insensitivity in wheat to a necrosis-inducing culture filtrate from Pyrenophora tritici-repentis. Phytopathology 86:459-463.

9. Faris, J. D., Haen, K. M., and Gill, B. S. 2000. Saturation mapping of a gene-rich recombination hot spot region in wheat. Genetics 154:823-835.

10. Feng, J., Ma, H., and Hughes, G. R. 2004. Genetics of resistance to Stagonospora nodorum blotch of hexaploid wheat. Crop Sci. 44:20432048.

11. Friesen, T. L., Meinhardt, S. W., and Faris, J. D. 2007. The Stagonospora nodorum-wheat pathosystem involves multiple proteinaceous hostselective toxins and corresponding host sensitivity genes that interact in an inverse gene-for-gene manner. Plant J. 51:681-692.

12. Friesen, T. L., Rasmussen, J. B., Kwon, C. Y., Ali, S., Francl, L. J., and Meinhardt, S. W. 2002. Reaction of Ptr ToxA-insensitive wheat mutants to Pyrenophora tritici-repentis race 1. Phytopathology 92:38-42.

13. Friesen, T. L., Stukenbrock, E. H., Liu, Z. H., Meinhardt, S. W., Ling, H., Faris, J. D., Rasmussen, J. B., Solomon, P. S., McDonald, B. A., and Oliver, R. P. 2006. Emergence of a new disease as a result of interspecific virulence gene transfer. Nat. Genet. 38:953-956.

14. Friesen, T. L., Zhang, Z., Solomon, P. S., Oliver, R. P., and Faris, J. D. 2008. Genetic characterization of a novel wheat-Stagonospora nodorum host-selective toxin interaction and its role in disease susceptibility. Plant Physiol. 146:682-693.

15. Gonzalez-Hernandez, J. L., Singh, P. K., Mergoum, M., Adhikari, T. B., Kianian, S. F., Simsek, S., and Elias, E. 2008. A quantitative trait locus on chromosome 5B controls resistance of Triticum turgidum (L.) var. dicoccoides to Stagonospora nodorum blotch. Euphytica DOI 10.1007/s10681-008-9825-z.

16. Haen, K. M., Lu, H.-J., Friesen, T. L., and Faris, J. D. 2004. Genomic targeting and high-resolution mapping of the Tsn1 gene in wheat. Crop Sci. 44:951-962.

17. Joehanes, R., and Nelson, J. C. 2008. QGene 4.0, an extensible Java QTLanalysis platform. Bioinformatics DOI: 10.1093/bioinformatics/btn523.

18. Joppa, L. R. 1993. Chromosome engineering in tetraploid wheat. Crop Sci. 33:908-913.

19. King, J. E., Cook, R. J., and Melville, S. C. 1983. A review of the Septoria diseases of wheat and barley. Ann. Appl. Biol. 103:345-374.

20. Kumar, S., Gill, B. S., and Faris, J. D. 2007. Identification and characterization of segregation distortion loci along chromosome $5 \mathrm{~B}$ in tetraploid wheat. Mol. Genet. Genomics 278:187-196.

21. Lamari, L., and Bernier, C. C. 1989. Toxin of Pyrenophora triticirepentis: host-specificity significance in disease, and inheritance of host reaction. Phytopathology 79:740-744.

22. Lamari, L., and Bernier, C. C. 1989. Virulence of isolates of Pyrenophora tritici-repentis on 11 wheat cultivars and cytology of the differential host reactions. Can. J. Plant Pathol. 11:284-290.

23. Lamari, L., and Bernier, C. C. 1991. Genetics of tan necrosis and extensive chlorosis in tan spot of wheat caused by Pyrenophora triticirepentis. Phytopathology 81:1092-1095.

24. Lander, E. S., Green, P., Abranhamson, J., Barlow, A., Daly, M. J., Lincoln, S. E., and Newburg, L. 1987. Mapmaker: an interactive computer package for constructing primary genetic linkage maps of experimental and natural populations. Genomics 1:174-181.

25. Liu, Z. H., Faris, J. D., Meinhardt, S. W., Ali, S., Rasmussen, J. B., and Friesen, T. L. 2004. Genetic and physical mapping of a gene conditioning sensitivity in wheat to a partially purified host-selective toxin produced by Stagonospora nodorum. Phytopathology 94:1056-1060.

26. Liu, Z. H., Friesen, T. L., Ling, H., Meinhardt, S. W., Oliver, R. P., Rasmussen, J. B., and Faris, J. D. 2006. The Tsn1-ToxA interaction in the wheat-Stagonospora nodorum pathosystem parallels that of the wheat-tan spot system. Genome 49:1265-1273.

27. Liu, Z. H., Friesen, T. L., Meinhardt, S. W., Ali, S., Rasmussen, J. B., and Faris, J. D. 2004. Quantitative trait loci analysis and mapping of seedling resistance to Stagonospora nodorum leaf blotch in wheat. Phytopathology 94:1061-1067.

28. Lu, H. J., and Faris, J. D. 2006. Macro- and micro-colinearity between the 
genomic region of wheat chromosome 5B containing the Tsn1 gene and the rice genome. Funct. Integr. Genomics 6:90-103.

29. Lu, H. J., Fellers, J. P., Friesen, T. L., Meinhardt, S. W., and Faris, J. D. 2006. Genomic analysis and marker development for the Tsnl locus using bin-mapped ESTs and flanking BAC contigs. Theor. Appl. Genet. 112:1132-1142.

30. Manly, K. F., Cudmore, R. H., Jr., and Meer, J. M. 2001. Map Manager QTX, cross platform software for genetic mapping. Mam. Gen. 12:930932.

31. Nelson, J. C. 1997. QGENE: Software for marker-based genomic analysis and breeding. Mol. Breed. 3:239-245.

32. Simons, K. J., Fellers, J. P., Trick, H. N., Zhang, Z. C., Tai, Y.-S., Gill, B. S., and Faris, J. D. 2006. Molecular characterization of the major wheat domestication gene $Q$. Genetics 172:547-555.

33. Tomás, A., and Bockus, W. W. 1987. Cultivar specific toxicity of culture filtrate of Pyrenophora tritici-repentis. Phytopathology 77:1337-1366.

34. Tomas, A., Feng, G. H., Reeck, G. R., Bockus, W. W., and Leach, J. E. 1990. Purification of a cultivar-specific toxin from Pyrenophora triticirepentis, causal agent of tan spot of wheat. Mol. Plant-Microbe Interact. 3:221-224.

35. Xu, S. S., Friesen, T. L., and Cai, X. W. 2004. Sources and genetic control of resistance to Stagonospora nodorum blotch in wheat. Recent Res. Dev. Genet. Breed. 1:449-469.

36. Zhang, H., Francl, L. J., Jordahl, J. G., and Meinhardt, S. W. 1997. Structural and physical properties of a necrosis-inducing toxin from Pyrenophora tritici-repentis. Phytopathology 87:154-160.

37. Zhang, Z., Friesen, T. L., Simons, K. J., Xu, S. S., and Faris, J. D. 2008. Development, identification, and validation of markers for markerassisted selection against the Stagonospora nodorum toxin sensitivity genes Tsn1 and Snn2 in wheat. Mol. Breed. 23:35-49. 